\title{
A Growth Model with Qualities, Varieties, and Human Capital: Stability and Transitional Dynamics
}

Tiago Neves Sequeira ${ }^{1}$, Alexandra Ferreira Lopes ${ }^{2}$, Orlando Gomes $^{3}$

${ }^{1}$ Universidade da Beira Interior, CEFAGE-UBI and INOVA

${ }^{2}$ ISCTE - IUL, ISCTE Business School, UNIDE - IUL and CEFAGE-UBI

${ }^{3}$ ISCAL - Lisbon Polytechnic Institute and UNIDE - IUL 


\title{
A Growth Model with Qualities, Varieties, and Human Capital: Stability and Transitional Dynamics*
}

\author{
Tiago Neves Sequeira ${ }^{\dagger} \quad$ Alexandra Ferreira Lopes ${ }^{\ddagger}$ \\ Orlando Gomes $\S$
}

\begin{abstract}
This article analyses the stability properties of the steady-state and the transitional dynamics of an endogenous growth model with human capital, increasing-varieties R\&D, and quality-ladders R\&D [Strulik, 2005, Review of International Economics, 13 (1): 129-145]. We show that when spillovers within $R \& D$ sectors are higher than spillovers across the two R\&D sectors, the equilibrium is unstable. However, when spillovers between sectors are higher than within, the equilibrium is a saddle-path. This result emphasizes the need for empirical research that compares quantitatively the importance of these two types of spillovers and highlights the importance of studying intersectoral effects between the two R\&D sectors. We describe plausible paths of economic development after changes in crucial parameters and uncover some transitional effects that were impossible to detect in the steady-state analysis. We also show that this model's transition dynamics can mimic the main features of the process of productivity slowdown that began in the 1970's.
\end{abstract}

JEL Classification: O11, O15, O31, O33, O41.

Keywords: Vertical and Horizontal R\&D, Human Capital, Endogenous Growth Models; Steady-State Stability; Transitional Dynamics.

*Tiago Neves Sequeira and Alexandra Ferreira Lopes acknowledge financial support from PTDC/EGE-ECO 102238/2008/FCT - Fundação para a Ciência e Tecnologia and Orlando Gomes acknowledges financial support from PEst-OE/EGE/UI0315/2011. The authors are also grateful to Manuel A. Gómez, for the permission to adapt some of the codes developed elsewhere to the numerical study of stability and to the transitional dynamics. The usual disclaimer applies.

†Universidade da Beira Interior, CEFAGE-UBI research center, and INOVA research center. E-mail: sequeira@ubi.pt.

${ }^{\ddagger}$ ISCTE - IUL (University Institute of Lisbon), ISCTE Business School Economics Department, UNIDE - IUL (BRU - Business Research Unit), and CEFAGE-UBI. E-mail: alexandra.ferreira.lopes@iscte.pt.

§ISCAL - Lisbon Polytechnic Institute and UNIDE - IUL (BRU - Business Research Unit). E-mail: omgomes@iscal.ipl.pt. 


\section{Introduction}

Strulik (2005) has been the first author to include increasing-varieties R\&D, qualityladders R\&D, and human capital accumulation in a model, constituting an important benchmark to study the relationships between the two well-known types of $\mathrm{R} \& \mathrm{D}$, in an environment where human capital accumulation is also considered. This model is an important contribution as a tool to study real-word mechanisms of innovation and human capital accumulation. Additionally, it also allows to have different effects of the population growth rate in economic growth, also considering different degrees of altruism. Strulik (2007) uses the same model and compares the market and the efficient equilibria, focusing on the optimality of investments in R\&D.

In Strulik (2005) the author described the steady-state features of the model. The stability analysis of the steady-state and transitional dynamics in such model is still to be done. This analysis is crucial to evaluate if the model steady-state is stable in the sense that some small change within the economic environment will make the economy to definitively depart from that equilibrium or, on the contrary, will converge to it again. We intend to fulfil this gap in the literature. To this end, we derive the equations that describe the dynamics of the Strulik (2005) model and then we show that there are possible combinations of parameters allowing for stability in the model. Meanwhile, we show that the combination of parameters presented in Strulik (2007) indeed yields an unstable equilibrium, inducing flawed interpretations. Then, the transitional dynamics of the model is characterized in detail, after some changes in crucial parameters.

Previously, an endogenous growth literature considering vertical and horizontal $\mathrm{R} \& \mathrm{D}$ emerged to face the counterfactual prediction of endogenous growth according to which growth is dependent on the size of the population (e.g. Grossman and Helpman, 1991; Aghion and Howitt, 1992) and the debatable prediction of semi-endogenous growth, according to which growth is solely dependent on the growth rate of the population (e.g., Jones, 1995). In fact, Dinopolous and Thompson (1998), Peretto (1998), and Young (1998) considered models with both types of R\&D activities and they obtained that growth is dependent on endogenous (without scale-effects) and semi-endogenous separate components. However, these models assumed that there are spillovers in vertical $R \& D$ and they are absent in horizontal R\&D. Li (2000) points out that the result according to which the output growth rate depends partially on the growth rate of population and partially on an nonscale endogenous component is dependent on a knife-edge condition that combines spillovers from both R\&D sectors. Generally, Li (2000) shows that besides this knife-edge condition, output growth can be endogenous growth with scale effects or semi-endogenous growth, totally dependent on the growth rate of population. In parallel with this literature, another stretch of literature introduced human capital in endogenous growth models with R\&D, beginning with Arnold (1998, 2000a). In a combination of both lines of research, Strulik (2005) includes human capital accumulation in the Li (2000)'s model. His model shows that growth is dependent on both a non-scale endogenous component and a semi-endogenous component depen- 
dent on population growth. However, this last part includes different possibilities for the sign of the correlation between growth and population growth, dependent on the degree of altruism.

One of the most recent attempts of endogenous growth theory is to study the stability of the steady-states and to use the transitional dynamics of the models to evaluate their fitness to reality (examples are Arnold, 2000b; Eicher and Turnovsky, 2001; Gómez, 2005; Gómez and Sequeira, 2011, for models with one R\&D sector). We pursue these lines of research in a model with human capital accumulation and both vertical and horizontal R\&D. We will show that one of the crucial issues regarding stability is the relationship between the two sectors of $\mathrm{R} \& \mathrm{D}$. Thus, our work opens interesting paths for future theoretical and empirical research. On the theoretical part, this is essential to evaluate the impact of fiscal policies on growth and welfare. On the empirical part, this shows the importance of assessing the intersectoral spillovers between R\&D sectors.

In the next section we present a short description of the Strulik (2005) model, presenting the most relevant equations for our analysis, and the stationary variables that allow us to derive the differential equations that describe the dynamics of the model. In section 3 we present the steady-state calculations for the stationary variables and a sensitivity analysis that shows the combination of parameters that enables the steady-state to be stable. Section 4 presents the transitional dynamics of the main variables in the model after some sensible changes in crucial parameters and also an exercise that shows that the model can mimic several empirical features of the productivity slowdown phenomenon after the seventies of the twentieth century. Section 5 concludes.

\section{The Model}

This section presents the model in Strulik (2005) and develops it in order to describe the dynamics. We will not repeat all the setup presented in Strulik (2005), we will instead recover the relevant equations and their description in order to allow for a self-contained reading.

\subsection{Setup}

The $R \& D$ activity is described by an increasing-varieties sector, in which the accumulation function is given by: $\frac{\dot{n}}{n}=A n^{\beta_{1}-1} Q^{\beta_{2}-1} H_{n}^{1-\chi}$, where $\beta_{1}$ measures the typical spillover effect, $\beta_{2}$ is the cross-sector spillover from the quality sector to the varieties sector, $\chi$ measures the duplication effect, $n$ is the number of varieties in the economy, $Q$ is the aggregate level of quality, and $H_{n}$ is the part of human capital that is allocated to the horizontal $\mathrm{R} \& \mathrm{D}$ sector.

Firms in the increasing-varieties sector maximize their expected value (the number of varieties produced times its value) minus research cost $\dot{n} V_{n}-w H_{n}$. The free entry condition is: $V_{n} \dot{n}=w H_{n} \Rightarrow \frac{\dot{V_{n}}}{V_{n}}=\frac{\dot{w}}{w}-\beta_{1} \frac{\dot{n}}{n}-\left(\beta_{2}-1\right) \frac{\dot{Q}}{Q}+\chi \frac{\dot{H_{n}}}{H_{n}}$. This means 
that the value of an innovation times the number of innovations must be equal to the cost of producing these innovations.

The non-arbitrage condition states that: $r=\pi_{0_{i}} / V_{n}+\frac{\dot{V_{n}}}{V_{n}}-\mu \frac{V_{0_{i}}}{V_{n}}$, meaning that the interest rate plus the innovation rate of success times the value of an innovation must be equal to the instant profits from selling the respective technology and the valorization of the patent.

There is also a quality-ladders sector, such that quality increases follow the accumulation function: $\frac{\dot{Q}}{Q}=\left(\gamma^{\sigma-1}-1\right) A n^{\alpha_{1}-1} Q^{\alpha_{2}-1} H_{Q}^{1-\chi}$, where $\alpha_{1}$ measures the cross-sector spillovers from the varieties sector into the quality-ladder sector, and $\alpha_{2}$ is the spillover within the quality ladders sector. The parameter $\gamma$ measures the increase in quality within each quality sector and thus the term $\gamma^{\sigma-1}-1$ measures the creative destruction effect. The allocation of human capital to the vertical R\&D sector is $H_{Q}$.

Firms in the quality-ladder sector maximize expected value minus research cost: $\mu_{k_{j}} V_{k_{j}}-w H_{Q_{j}}$, where $\mu$ is the probability of success of a vertical innovation and $w$ is the wage of human capital. The free entry condition is: $V_{k_{j}} \dot{Q} n=w H_{Q_{j}} \Rightarrow$ $\frac{\dot{V_{k_{j}}}}{V_{k_{j}}}=\frac{\dot{w}}{w}-\alpha_{1} \frac{\dot{n}}{n}-\alpha_{2} \frac{\dot{Q}}{Q}+\chi \frac{\dot{H_{Q}}}{H_{Q}}$. This means that the value of the innovation times the number of innovations made on the whole available technologies must be equal to the cost of producing these innovations.

The non-arbitrage condition states that: $r=\pi_{k_{j}} / V_{k_{j}}+\frac{\dot{V_{k_{j}}}}{V_{k_{j}}}-\mu_{k_{j}}$, meaning that the interest rate plus the innovation rate of success times the value of an innovation must be equal to the instant profits from selling the respective technology and the valorization of the patent.

Let $g_{i}$ denote the growth rate of variable $i$. Households maximize intertemporal utility from consumption per capita: $c=Y / L: U_{t}=\int_{0}^{\infty} \frac{c^{1-\theta}}{1-\theta} e^{-\left(\rho-m g_{L}\right) t} d t$, where $L$ is population size. Population grows at a given constant rate, $g_{L}$, which may be positive, negative, or zero. The time preference rate is denoted by $\rho>0$, and $1 / \theta$ is the intertemporal elasticity of substitution. Concerning the parameter $m$, most of the literature considers one of the border cases. For $m=0$, households maximize utility of consumption per capita and so the utility function is of the Millian type. For $m=1$, the utility function is of the Benthamite type: households maximize utility of consumption of all members of their dynasty. Thus, as Strulik (2005) pointed out, $m$ controls for the degree of altruism towards future generations.

From the former equalities and arbitrage conditions in both R\&D sectors, the author reaches the following equations:

$$
\begin{aligned}
& \xi-\delta=\left(\frac{H_{X}}{H_{Q}} \frac{1}{\sigma-1}-1\right) \frac{\dot{Q}}{Q} \frac{1}{\gamma^{\sigma-1}-1}-\alpha_{1} \frac{\dot{n}}{n}-\alpha_{2} \frac{\dot{Q}}{Q}+\chi \frac{\dot{H}_{Q}}{H_{Q}} \\
& \xi-\delta=\left(\frac{1}{\sigma-1} \frac{H_{X}}{H_{n}}-\frac{H_{Q}}{H_{n}}\right) \frac{\dot{n}}{n}-\beta_{1} \frac{\dot{n}}{n}-\left(\beta_{2}-1\right) \frac{\dot{Q}}{Q}+\chi \frac{\dot{H}_{n}}{H_{n}}
\end{aligned}
$$

Additionally, from the first order conditions of the consumer problem, demand for 
human capital, and the production function, and taking into account the market clearing condition $C=Y$, the following equations are obtained:

$$
\begin{aligned}
& \frac{\dot{Y}}{Y}=\frac{1}{\theta}\left[\frac{1}{\sigma-1}\left(\frac{\dot{n}}{n}+\frac{\dot{Q}}{Q}\right)+\xi-\delta-\rho+m g_{L}\right] \\
& \dot{Y}=\frac{1}{\sigma-1}\left(\frac{\dot{n}}{n}+\frac{\dot{Q}}{Q}\right)+\frac{\dot{H}_{X}}{H_{X}}
\end{aligned}
$$

Finally, from the growth rate of human capital and the human capital resource constraint, we obtain $\frac{\dot{H}}{H}=\xi\left(1-\frac{H_{X}}{H}-\frac{H_{Q}}{H}-\frac{H_{n}}{H}\right)-\delta$.

\subsection{Dynamics}

We will solve the dynamics of the model taking into account the following five stationary variables:

$$
\begin{aligned}
v_{Q} & =\frac{Q}{H^{\frac{\beta_{1}-\alpha_{1}}{D}(1-\chi)}} ; \\
v_{n} & =\frac{n}{H^{\frac{\alpha_{2}-\beta_{2}}{D}(1-\chi)}} ; \\
\mu_{X} & =\frac{H_{X}}{H}, \mu_{Q}=\frac{H_{Q}}{H}, \mu_{n}=\frac{H_{n}}{H} .
\end{aligned}
$$

where $D=\left(1-\alpha_{1}\right)\left(1-\beta_{2}\right)-\left(1-\alpha_{2}\right)\left(1-\beta_{1}\right)$. The first two are state variables and the last three are control variables. Based on these variables, we will derive a system of five differential equations.

By the log-differentiation of (5), using the equations for the growth rate of qualities and the growth rate of human capital, given above, we obtain:

$$
\begin{gathered}
\frac{\dot{v_{Q}}}{v_{Q}}=\left(\gamma^{\sigma-1}-1\right) A v_{n}^{\alpha_{1}-1} v_{Q}^{\alpha_{2}-1} \mu_{Q}^{1-\chi}- \\
-\frac{\beta_{1}-\alpha_{1}}{D}(1-\chi)\left[\xi\left(1-\mu_{X}-\mu_{Q}-\mu_{n}\right)-\delta\right]
\end{gathered}
$$

By the log-differentiation of (6), using the equations for the growth rate of varieties and the growth rate of human capital given above we obtain:

$$
\begin{gathered}
\frac{\dot{v}_{n}}{v_{n}}=A v_{n}^{\beta_{1}-1} v_{Q}^{\beta_{2}-1} \mu_{n}^{1-\chi}- \\
\frac{\alpha_{2}-\beta_{2}}{D}(1-\chi)\left[\xi\left(1-\mu_{X}-\mu_{Q}-\mu_{n}\right)-\delta\right]
\end{gathered}
$$

By the log-differentiation of $\mu_{X}, \mu_{Q}$, and $\mu_{n}$, we obtain, respectively, the following dynamic equations. For the equation of $\mu_{X}$, we use (3) and (4) and the above 
law of motion for human capital. For the equation for $\mu_{Q}$ we use (1) and the law of motion for human capital. Finally for the equation for $\mu_{n}$ we resort to (2) and to the law of motion for human capital.

$$
\begin{aligned}
& \frac{\dot{\mu}_{X}}{\mu_{X}}=\frac{1-\theta}{\theta} \frac{1}{\sigma-1}\left[A v_{n}^{\beta_{1}-1} v_{Q}^{\beta_{2}-1} \mu_{n}^{1-\chi}+\left(\gamma^{\sigma-1}-1\right) A v_{n}^{\alpha_{1}-1} v_{Q}^{\alpha_{2}-1} \mu_{Q}^{1-\chi}\right] \\
& +\frac{1}{\theta}\left(\xi-\delta-\rho+m g_{L}\right)-\left[\xi\left(1-\mu_{X}-\mu_{Q}-\mu_{n}\right)-\delta\right] \\
& \frac{\dot{\mu}_{Q}}{\mu_{Q}}=\frac{1}{\chi}\left[\xi-\delta-\left(\frac{\mu_{X}}{\mu_{Q}} \frac{1}{\sigma-1}-1-\alpha_{2}\left(\gamma^{\sigma-1}-1\right)\right) A v_{n}^{\alpha_{1}-1} v_{Q}^{\alpha_{2}-1} \mu_{Q}^{1-\chi}+\alpha_{1} A v_{n}^{\beta_{1}-1} v_{Q}^{\beta_{2}-1} \mu_{n}^{1-\chi}\right] \\
& -\left[\xi\left(1-\mu_{X}-\mu_{Q}-\mu_{n}\right)-\delta\right] \\
& \frac{\dot{\mu}_{n}}{\mu_{n}}=\frac{1}{\chi}\left[\xi-\delta-\left(\frac{1}{\sigma-1} \frac{\mu_{X}}{\mu_{n}}-\frac{\mu_{Q}}{\mu_{n}}-\beta_{1}\right) A v_{n}^{\beta_{1}-1} v_{Q}^{\beta_{2}-1} \mu_{n}^{1-\chi}\right. \\
& \left.+\left(\beta_{2}-1\right)\left(\gamma^{\sigma-1}-1\right) A v_{n}^{\alpha_{1}-1} v_{Q}^{\alpha_{2}-1} \mu_{Q}^{1-\chi}\right]- \\
& -\left[\xi\left(1-\mu_{X}-\mu_{Q}-\mu_{n}\right)-\delta\right]
\end{aligned}
$$

\section{$3 \quad$ Steady-State and Stability Analysis}

Steady-State values for the five stationary variables were obtained as follows. We write equation (1) in order to $\mu_{X}$, where this variable is related to $\mu_{Q}$. Then from equation (2) we write $\mu_{n}$ as a function of $\mu_{Q}$. Substituting the expressions of $\mu_{n}$ and $\mu_{X}$ in $\frac{\dot{H}}{H}=\xi\left(1-\frac{H_{X}}{H}-\frac{H_{Q}}{H}-\frac{H_{n}}{H}\right)-\delta$, we obtain the steady-state equation for $\mu_{Q}$. Substituting the equation of $\mu_{Q}$ in the previous two we find, respectively, $\mu_{X}$ and $\mu_{n}$.

Equalizing equation (8) to equation (9) we encounter an equation for $v_{n}$ that depends on $v_{Q}, \mu_{Q}$, and $\mu_{n}$. Substituting the steady-state equations found for $\mu_{Q}$, and $\mu_{n}$, and substituting the entire equation for $v_{n}$ in (8) we arrive to the steadystate equation for $v_{Q}$. Finally, we substitute the equation for $v_{Q}$ in the equation for $v_{n}$ to find the steady-state equation for $v_{n}{ }^{1}$

The values for the stationary variables in the steady-state are positive if either $\alpha_{1}>\beta_{1}$ and $\alpha_{2}<\beta_{2}$ or $\alpha_{1}<\beta_{1}$ and $\alpha_{2}>\beta_{2}$. Additionally, the productivity on human capital accumulation must be sufficiently high, so that $\xi>$ $\delta+\frac{\left(\theta-(1-m) g_{L}\right)-\rho}{\left(\frac{(\theta-1)(1-\chi)}{\sigma-1}\right)\left(\frac{\beta_{1}-\alpha_{1}+\alpha_{2}-\beta_{2}}{D}\right)+(\theta-1)}$.

In order to analyze the local stability of the steady-state, we linearize the system of equations (8) to (12) around its steady-state $\left(v_{Q}^{*}, v_{n}^{*}, \mu_{X}^{*}, \mu_{Q}^{*}, \mu_{n}^{*}\right)$. This gives the following five-order system:

\footnotetext{
${ }^{1}$ Since equations representing the steady-state for each of this stationary variables are too long, we do not show them in the paper. They are available upon request.
} 


$$
\left(\begin{array}{c}
\dot{v_{Q}} \\
\dot{v_{n}} \\
\dot{\mu_{X}} \\
\dot{\mu_{Q}} \\
\mu_{n}
\end{array}\right)=\mathbf{J}\left(\begin{array}{c}
v_{Q}-v_{Q}^{*} \\
v_{n}-v_{n}^{*} \\
\mu_{X}-\mu_{X}^{*} \\
\mu_{Q}-\mu_{Q}^{*} \\
\mu_{n}-\mu_{n}^{*}
\end{array}\right),
$$

where $\mathbf{J}$ is presented in the Appendix.

There are two state-variables $v_{Q}$ and $v_{n}$ and three control variables, which are the shares of human capital allocated to the industrial sector and to both R\&D sectors $\left(\mu_{X}, \mu_{Q}, \mu_{n}\right)$. As in Eicher and Turnvosky (2001), that studied a four-dimension system with two state and two control variables, it is impossible to find a general condition that grants stability. In this case, however, it is also impossible to rule out cases of instability and indeterminacy, due to the dimension and the complexity of the Jacobian matrix. Thus, we pursue the analysis by presenting a sensitivity analysis for a grid of parameters that suggests that only for one precise combination of parameters linked with spillovers and cross-spillovers, it is possible for the steadystate to be saddle-path stable. First, the following example shows that for the combination of parameters used in Strulik (2007), the steady-state is unstable. This highlight the importance of studying the stability of the steady-state.

Example 1. The parameterization $\beta_{1}=1, \beta_{2}=0.69, \alpha_{1}=0.69, \alpha_{2}=1, \chi=0.5$, $\sigma=6.00, \xi=0.0675, \rho=0.02, \theta=2.45, \delta=0.01$ and $\gamma=1.1$ used in Strulik (2007) yields the (feasible) steady-state: $v_{Q}=252.0, v_{n}=63.99, \mu_{X}=0.501$, $\mu_{Q}=0.033, \mu_{n}=0.030, g_{Y}=0.0175$. The eigenvalues of the linearized system are $0.245513,0.0888441,0.0381291,-0.0163393$ and 0.0152407 and, therefore, the steady-state is unstable.

Next, we present a sensitivity analysis that is based on a grid exercise on $\beta_{1}$, $\beta_{2}, \alpha_{1}$, and $\alpha_{2}$, with step 0.025, considering all the other parameters as in Example 1 , except for $\xi$ that we also compute differently such that, in each case, it makes the growth rate of output $\left(g_{Y}\right)$ to be 0.0175 . For each case, we present the number of cases of stability (both with real and complex conjugate negative roots) and instability results.

We study both cases for which it is possible to have feasible steady-states - case 1: $\alpha_{1}<\beta_{1}$ and $\alpha_{2}>\beta_{2}$; case 2: $\alpha_{1}>\beta_{1}$ and $\alpha_{2}<\beta_{2}$. While case 1 assumes that spillovers within sectors are higher than spillovers between spillovers, case 2 states that spillovers between sectors are higher than spillovers within sectors. The first case means that varieties $R \& D$ depends more on the existence of different varieties and quality R\&D depends more on higher qualities; on the opposite, the second case means that varieties $R \& D$ depends more on higher qualities and qualities R\&D depends more on the existence of different varieties. Neither articles from Strulik $(2005,2007)$ argue for the most realistic case, although Strulik (2007) has considered an extreme case of self-spillovers equal to 1 and cross-spillovers equal to 0.69 (specific values for case 1).

For each case we present five exercises. In the first exercise, we fixed the values for the spillovers in the quality-ladders sector and kept other spillovers free. In 
the second exercise, we fixed values for spillovers in the varieties sector, setting a value for spillovers within varieties sector equal to 0.8 , a value close to that used in previous literature (see Jones and Williams, 2000 and Funke and Strulik, 2000). In the third and fourth exercises, we just fix values for the spillover within the varieties sector in two values: 0.8 and 0.4 , which have been used in different articles (see Reis and Sequeira, 2007). Finally we also include an exercise in which the spillover in horizontal R\&D is set to 0.2 . Results are in Table 1 below.

Table 1: Number of Stable and Unstable Cases for Intervals of Spillovers Values

\begin{tabular}{cccccc}
\hline \hline & $\alpha_{1}=0.69 ; \alpha_{2}=1$ & $\beta_{1}=0.8 ; \beta_{2}=0.69$ & $\beta_{1}=0.8$ & $\beta_{1}=0.4$ & $\beta_{1}=0.2$ \\
\hline Stable (real) & 0 & $\alpha_{1}<\beta_{1}$ and $\alpha_{2}>\beta_{2}$ \\
Stable (complex) & 0 & 0 & 0 & 0 & 0 \\
Unstable & 508 & 0 & 0 & 0 & 0 \\
\hline & 412 & 25488 & 12616 & 6286 \\
\hline Stable (real) & - & $\alpha_{1}>\beta_{1}$ and $\alpha_{2}<\beta_{2}$ \\
Stable (complex) & - & 224 & 6181 & 18797 & 25488 \\
Unstable & - & 0 & 95 & 223 & 226 \\
\hline \hline
\end{tabular}

The first important result driven from the analysis of our results is that indeterminacy may be a negligible result, as in our grid analysis there were no cases of indeterminacy. More important than that, the values in Table 1 suggest a strong result according to which feasible steady-state are only saddle-path stable when spillovers between sectors are higher than spillovers within sectors. Moreover, in this case, the transition to the steady-state may, in most circumstances, be monotonic or in a minority of cases, be oscillatory. In fact, complex conjugate eigenvalues appear in less than $2 \%$ of all the stable cases.

This result emphasizes the need for empirical research that compares quantitatively the importance of these two types of spillovers and highlights the importance of studying intersectoral effects between the two R\&D sectors.

\section{Transitional Dynamics}

\subsection{Transitional Dynamics after Changes in Parameters}

In this section we present the transitional dynamics of this economy after changes in some crucial parameters. In particular, we display the evolution of the economy after a decrease in the growth rate of the labor force from $1.44 \%$ to $0.72 \%$ (halving the population growth rate) and also after an increase in productivity of the human capital accumulation sector from 0.0675 to 0.1 . These exercises show the importance of studying the transitional dynamics in the evolution of variables. We consider the following set of initial parameters that guarantee that the equilibrium is saddle-path stable: $\beta_{1}=0.25, \beta_{2}=0.25, \alpha_{1}=0.4, \alpha_{2}=0.1, \chi=0.5, \sigma=6.00, \xi=0.0675$, 

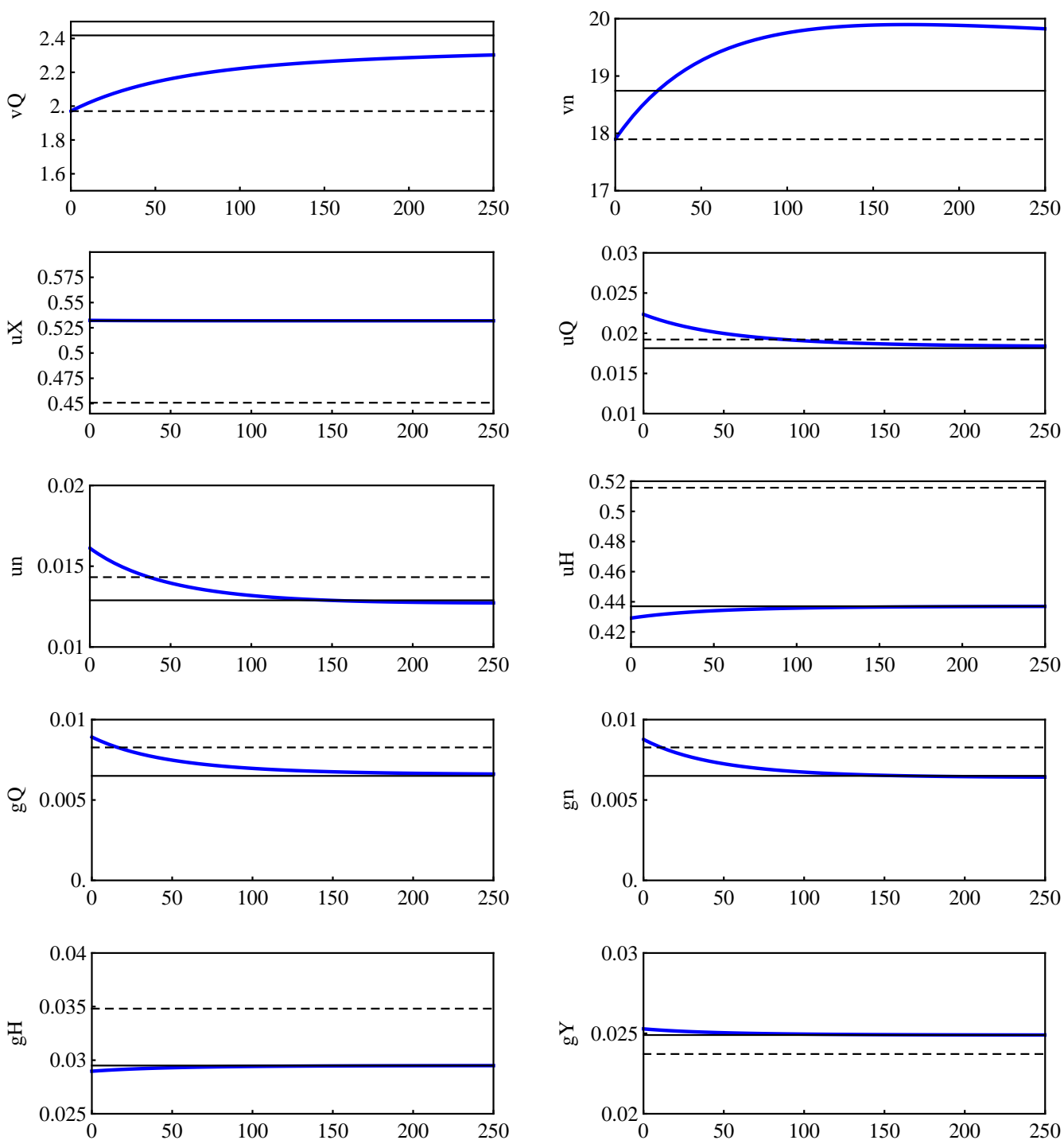

Figure 1: Adjustment paths of main variables after a change in $g_{L}$ from 0.0144 to 0.0072. Note: dashed black lines indicates the initial steady-state and solid black line indicates the final steady-state. 
$\rho=0.02, \theta=2.45, \delta=0.01$, and $\gamma=1.1$. We use the relaxation method due to Trimborn et al. (2008).

In Figure 1 we can observe the evolution of the economy after halving the population growth rate. This implies a lengthy transition path of the state variables that measure the relative importance of R\&D (both types) when compared to human capital. As population growth decreases there is lesser need for faster human capital accumulation. This induces a trade-off between human capital and R\&D which favors the last. Because of that, Figure 1 shows a decrease of investment in human capital (both the evolution of its share $\mu_{H}$ and its growth rate $g_{H}$ demonstrate this effect) and an increase in the shares and growth rates of $R \& D$ (both in the qualities and varieties sectors). An interesting effect hidden by the steady-state analysis is the short-run overshooting of R\&D which amounts to near $0.25 \%$ of human capital allocated to each of the R\&D sectors. This is compensated by an initial undershooting of the human capital allocated to the human capital accumulation sector. The transitional dynamics of the varieties sector is also worth noting. In fact, while it initially overshoots, nearly 150 years after the decrease in population growth rate it passes below its long run value, representing a slow decrease in the share of the horizontal R\&D sector. The negative correlation between the growth rate of population and economic growth is seen in the last panel of Figure 1 and it is underestimated by a simple steady-state analysis. Moreover we can observe that a sudden decrease in the population growth rate induces a fast increase in industry - see the panel for $\mu_{X}$, in which the drop in population growth rate implied an increase of near $7.5 \%$ in human capital allocated to that sector. This is obtained at the expense of decreases in the long-run values of $R \& D$ and human capital, despite the initial increase in both $R \& D$ sectors. These are the predictions that these model carries out for countries that experience a sudden drop in the population growth (e.g. China).

Figure 2 shows the evolution of the economy after an increase in the productivity of the human capital accumulation sector. This increases the importance of human capital in the economy, decreasing the importance of $v_{n}$ and $v_{Q}$. The consequence is a decrease in the allocation of human capital to human capital accumulation, and also for the qualities and varieties sector. While the drop on the share of human capital allocated to schools (i.e. , in its own accumulation) is permanent, the drop of human capital allocated to the R\&D sectors is only an initial effect that is evident in the first 50 years of transition. Initially, shares of human capital drop by more than $1 \%$ in both research sectors, which represents nearly $30 \%$ of the steady-state value for these variables. Growth rates of $R \& D$ decrease from their initial values in the first 10 years and then slowly increase towards their long-run values. The effect on the economic growth rate is nearly $2 \%$ and it jumps initially almost the entire difference.

\subsection{Transitional Dynamics mimics Productivity Slowdown}

One of the main features of the evolution of the economy after the 1970 decade was the productivity slowdown in most developed countries, a process documented by 

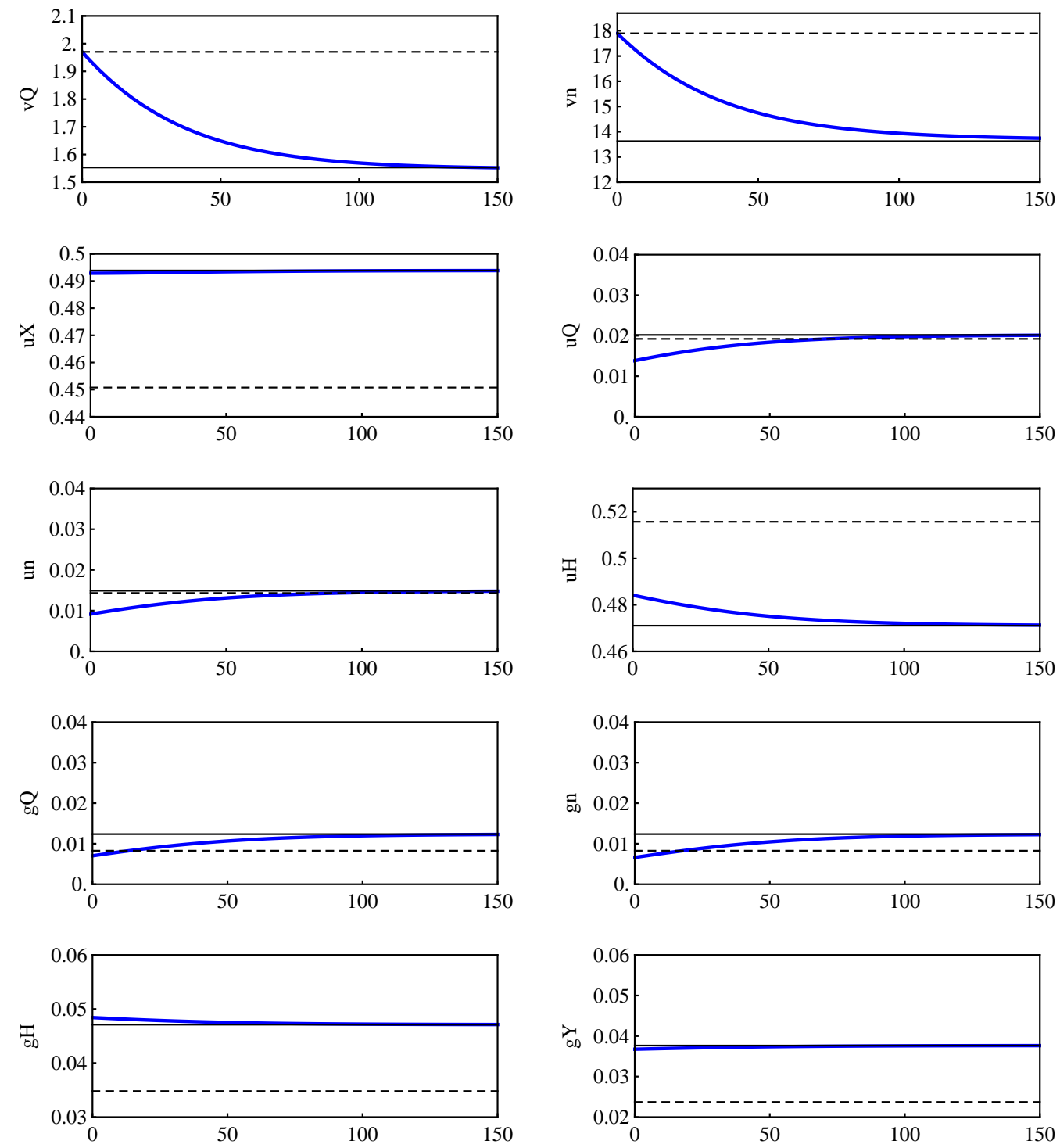

Figure 2: Adjustment paths of main variables after a change in $\xi$ from 0.0675 to 0.1. Note: dashed black lines indicates the initial steady-state and solid black line indicates the final steady-state. 
Saltari and Travaglini (2009). Simultaneously, there has been a huge investment in education with average years of education doubling in some developed countries. However, growth in human capital was insufficient to compensate the huge drop in productivity (TFP) and GDP per capita growth also decreased a lot. Table 2 shows examples of the data that supports those findings. We use data for EU15 and France but several developed countries present a similar path.

Table 2: The Technological Slowdown and Education Rise

\begin{tabular}{ccccc}
\hline \hline & $1971-80$ & $1981-1990$ & $1990-1994$ & $1995-2007$ \\
\hline GDP per hour growth (EU15) & $3.8 \%$ & $2.3 \%$ & $2.4 \%$ & $1.4 \%$ \\
TFP growth (France) & $2.1 \%$ & \multicolumn{2}{c}{$1.5 \%$} & $1.0 \%$ \\
Attainment (France) & 5.49 & 6.73 & 8.02 & 9.34 \\
Sec. Enrollment (France) & 7.5 & 15.8 & 25.3 & 33 \\
\hline \multicolumn{5}{c}{ Sources: Saltari and Travaglini (2009) and Barro and Lee (2010) } \\
Note: In Education data, averages were used to close match the periods for GDP and TFP \\
\hline \hline
\end{tabular}

In this section we show that this model closely matches this evolution. We set $\xi$ so as GDP per capita matches the $1.4 \%$ growth rate of the EU15 between 1995 and 2007 and population growth is also set to $1.2 \%$, according to the growth rate of employment in EU15 in the same period, reported in Saltari and Travaglini (2009). Figure 3 shows an economy in which GDP per capita decreases a lot in 40 years from around $4 \%$ to near $1.4 \%$. This was exactly what happened in most developed countries and what happened in EU15, as Table 2 reports. The model predicts a huge decrease in TFP, from $6 \%$ to near $0.5 \%$, even more than the slowdown occurred in France. In Spain and Italy, for instance, TFP growth had dropped nearly 13 times from $2.6 \%$ to near $0.2 \%$, with the same period. The simulation highlights an interesting pattern, the fact that the drop in TFP growth rate is mainly due an huge drop in the growth rate of the number (variety) of technologies and not so much due to the decrease in the technologies quality, meaning that after some periods the average quality per technology is rising (as quality is growing more than varieties), which apparently has also occurred in the real world. This exercise also shows that the drop in TFP is consistent with a systematic increase in human capital. In fact human capital growth begins near $1.3 \%$ and rises to $2 \%$, growth rates comparable to the ones implied by the values in the Table to France (they are around $1.5 \%$ per year if we consider Attainment and $3 \%$ per year if we consider enrollment). We note that enrollment rates cannot be directly compared with our variable $u_{H}$ as this can include home education and other types of out-of-the-market learning. However, Sweden had 33.6\% secondary enrollment in 1971-80 and 47.6\% in 1995-2007, very similar values to those in Figure 3.

This exercise shows that, even though the model is quite stylized and does not incorporate some important issues, such as physical capital accumulation, it has interesting properties that can make it an interesting benchmark model to mimic the evolution of the economy. 

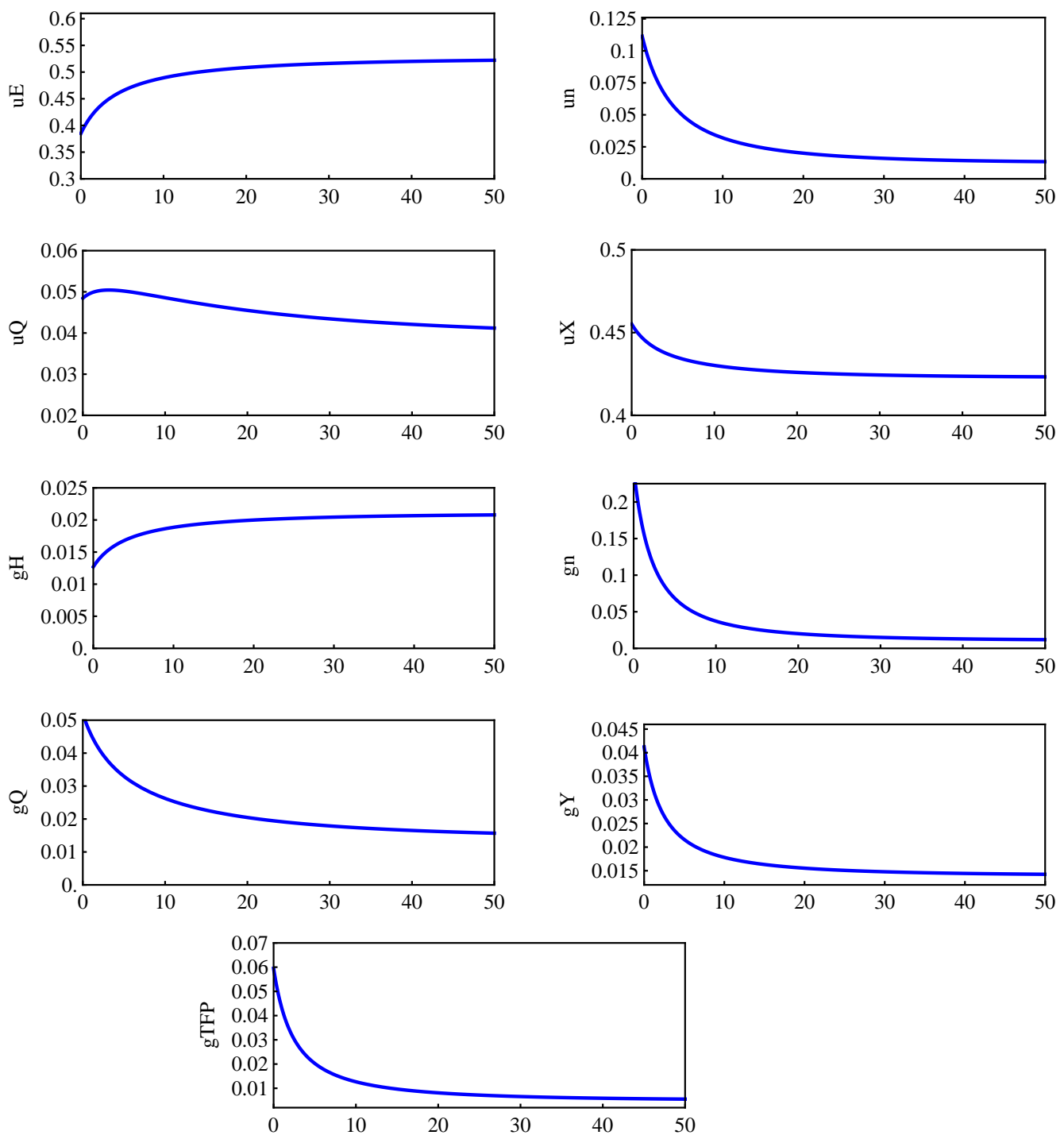

Figure 3: Adjustment paths of main variables that mimics Productivity Slowdown. 


\section{Conclusions}

We study the transitional dynamics of a model of endogenous growth, which includes human capital accumulation, vertical, and also horizontal R\&D (due to Strulik, 2005). It features a non-scale economic growth rate that can depend positively or negatively on the growth rate of the population, where the author has studied the steady-state features of the model. This article complements his analysis in two directions. First, we study the stability properties of the equilibrium manifold. Second, we studied transitional dynamics trajectories. Interestingly, we have concluded that stability is not guaranteed in this model and in fact, every studied solutions in which spillovers within R\&D sectors are higher than spillovers between R\&D sectors (as the examples in Strulik, 2007) are unstable, thus yielding least interesting conclusions. Moreover, we conclude that the probability of encompassing saddle-path stability is very high when spillovers between R\&D sectors are higher than spillovers within R\&D sectors. This result emphasizes the need for empirical research that compares quantitatively the importance of these two types of spillovers and highlights the importance of studying intersectoral effects between the two R\&D sectors.

We showed transitional dynamics after changes in the growth rate of population and changes in the productivity of the human capital accumulation sector. This analysis highlights transitional effects that were hidden by the steady-state analysis. In particular, we discovered significant overshooting and undershooting effects on $R \& D$ variables, when we perform changes in the growth rate of the population and changes in the productivity of the human capital accumulation sector. Moreover, we showed that the model can mimic the most important features of the productivity slowdown process that began in the 1970's.

As the knowledge of the transitional dynamics of a growth model is essential for the evaluation of its adherence to reality and to correctly evaluate the introduction of economic policies in the theoretical economy, the use of this model to study these issues are possible avenues of future research.

\section{References}

[1] Aghion, P. and P. Howitt (1992), "A Model of Growth through Creative Destruction", Econometrica 60 (2): 323-351.

[2] Arnold, L. (1998), "Growth, Welfare and Trade in an Integrated Model of Human-Capital Accumulation and Research", Journal of Macroeconomics 20 (1): 81-105.

[3] Arnold, L. (2000a), "Endogenous Growth with Physical Capital, Human Capital and Product Variety: A Comment", European Economic Review 44: 15991605.

[4] Arnold, L. (2000b), "Endogenous Technological Change: A Note on Stability", Economic Theory 16: 219-226. 
[5] Dinopoulos, E. and P. Thompson (1998), "Schumpeterian Growth Without Scale Effects", Journal of Economic Growth 3: 313-35.

[6] Barro, R. and J-W. Lee, April 2010, "A New Data Set of Educational Attainment in the World, 1950-2010." NBER Working Paper No. 15902.

[7] Eicher, T. and S. Turnovsky (2001), "Transitional Dynamics in a Two-Sector Non-Scale Growth Model", Journal of Economic Dynamics and Control 25: 85-113.

[8] Funke, M. and H. Strulik (2000), "On Endogenous Growth with Physical Capital, Human Capital and Product Variety", European Economic Review 44: 491-515.

[9] Gómez, M. (2005), "Transitional Dynamics in an Endogenous Growth Model with Physical Capital, Human Capital and R\&D", Studies in Nonlinear Dynamics and Econometrics 9 (1), Article 5.

[10] Gómez, M. and T. N. Sequeira (2011), "The Transitional Dynamics of an Endogenous Growth Model: Generalizing Production Functions", Studies in Nonlinear Dynamics and Econometrics, forthcoming.

[11] Grossman, G. and E. Helpman (1991), Innovation and Growth in the Global Economy, MIT Press.

[12] Jones, C. I. (1995), "R\&D-Based Models of Endogenous Growth", Journal of Political Economy 103 (4): 759-584.

[13] Jones, C. I. and J. Williams (2000), "Too Much of a Good Thing? The Economics of Investment in R\&D", Journal of Economic Growth 5: 65-85.

[14] Li, C. W. (2000), "Endogenous vs. Semi-endogenous Growth in a Two R\&Dsector Model", Economic Journal 110: C109-122.

[15] Peretto, P. F. (1998), "Technological Change and Population Growth", Journal of Economic Growth 3: 283-311.

[16] Reis, A. B. and T. N. Sequeira (2007), "Human Capital and Overinvestment in R\&D", Scandinavian Journal of Economics 109 (3): 573-591.

[17] Strulik, H. (2005), "The Role of Human Capital and Population Growth in R\&D-Based Models of Economic Growth", Review of International Economics 13 (1): 129-145.

[18] Strulik, H. (2007), "Too Much of a Good Thing? The Quantitative Economics of R\&D Driven Growth Revisited", Scandinavian Journal of Economics 109 (2): 369-386. 
[19] Saltari, E. and G. Travaglini (2009), "The Productivity Slowdown Puzzle. Technological and Non-technological Shocks in the Labor Market", International Economic Journal, 23 (4), 483-509.

[20] Trimborn, T, K. Koch, and T. Steger (2008), "Multidimensional Transitional Dynamics: A Simple Numerical Procedure", Macroeconomic Dynamics 12 (3): 301-319.

[21] Young, A. (1998), "Growth Without Scale Effects," Journal of Political Economy 106: 41-63. 


\section{Appendix: Jacobian}

$\mathbf{J}=\left(\begin{array}{ccccc}j_{11} & \frac{1-\alpha_{1}}{1-\alpha_{2}} \frac{j_{11}}{v_{n}} & \frac{v_{Q} \xi(1-\chi)\left(\beta_{1}-\alpha_{1}\right)}{D} & -v_{Q}(1-\chi)\left(\frac{j_{11}}{\mu_{Q}}+\frac{\xi\left(\beta_{1}-\alpha_{1}\right)}{D}\right) & \frac{v_{Q} \xi(1-\chi)\left(\beta_{1}-\alpha_{1}\right)}{D} \\ j_{21} & \frac{1-\beta_{1}}{1-\beta_{2}} j_{21} v_{Q} & \frac{v_{n} \xi(1-\chi)\left(\beta_{2}-\alpha_{2}\right)}{D} & \frac{v_{n} \xi(1-\chi)\left(\beta_{2}-\alpha_{2}\right)}{D} & j_{25} \\ j_{31} & j_{32} & \xi \mu_{X} & j_{34} & j_{35} \\ j_{41} & j_{42} & j_{43} & j_{44} & j_{45} \\ j_{51} & j_{52} & j_{53} & j_{54} & j_{55}\end{array}\right)$,

$$
\begin{aligned}
& j_{11}=-A\left(1-\alpha_{2}\right)\left(\gamma^{-1+\sigma}-1\right) \mu_{Q}^{1-\chi} v_{n}^{-1+\alpha_{1}} v_{Q}^{-1+\alpha_{2}} \text {; } \\
& j_{21}=-A\left(1-\beta_{2}\right) \mu_{n}^{1-\chi} v_{n}^{\beta_{1}} v_{Q}^{-2+\beta_{2}} \text {; } \\
& j_{31}=\frac{A \mu_{X}(1-\theta)\left(\left(\mu_{Q}^{1-\chi} V^{\boldsymbol{\alpha}}\left(\gamma^{-1+\sigma}-1\right)\left(1-\alpha_{2}\right)+\mu_{n}^{1-\chi} V^{\boldsymbol{\beta}}\left(1-\beta_{2}\right)\right)\right.}{V v_{Q} \theta(\sigma-1)} ; \\
& j_{41}=\frac{A u_{Q}\left(\left(1-\alpha_{2}\right) \mu_{Q}^{1-\chi} V^{\alpha}\left(\frac{\mu_{X}}{\mu_{Q}(\sigma-1)}+\left(1-\gamma^{-1+\sigma}\right) \alpha_{2}-1\right)-\mu_{n}^{1-\chi} V^{\boldsymbol{\beta}}\left(1-\beta_{2}\right)\right)}{V v_{Q} \chi} ; \\
& j_{51}=\frac{A \mu_{n}\left(\mu_{Q}^{1-\chi} V^{\boldsymbol{\alpha}}\left(1-\alpha_{2}\right)\left(1-\gamma^{-1+\sigma}\right)+\frac{\mu_{n}^{-\chi} V^{\boldsymbol{\beta}}\left((1-\sigma) \mu_{Q}+\mu_{X}+\mu_{n}(1-\sigma) \beta_{1}\right.}{(1-\sigma)}\right)\left(\beta_{2}-1\right)}{V v_{Q} \chi} ; \\
& j_{43}=\mu_{Q}\left(\xi+\frac{\mu_{Q} j_{11}}{\chi(1-\sigma)\left(1-\alpha_{2}\right)\left(\gamma^{-1+\sigma}-1\right)}\right) ; \\
& j_{53}=\mu_{n}\left(\xi+\frac{A \frac{\mu_{n} v_{Q}}{v_{n}} j_{21}}{\chi(1-\sigma)\left(1-\beta_{2}\right)}\right) ; \\
& j_{34}=\mu_{X}\left(\xi+\frac{j_{11}}{\mu_{Q}\left(\gamma^{-1+\sigma}-1\right) \gamma \theta(1-\sigma)\left(1-\alpha_{2}\right)}\right) ; \\
& j_{44}=\mu_{Q}\left(\xi+\frac{j_{11}\left(A \gamma\left(\chi \mu_{X}-\mu_{Q}(1-\sigma)(1-\chi)+A \alpha_{2} \mu_{Q}(1-\chi)(1-\sigma)\left(-\gamma+\gamma^{\sigma}\right)\right)\right.}{\mu_{Q}^{2}\left(\gamma^{-1+\sigma}-1\right) \gamma(1-\sigma)\left(1-\alpha_{2}\right)}\right) \text {; } \\
& j_{54}=\mu_{n}\left(\xi+\frac{A\left[\left(\frac{j_{21}}{\left(1-\beta_{2}\right)}\right)+\left(\frac{j_{11} v_{n}(1-\chi)}{\left(1-\alpha_{2}\right)\left(\gamma^{-1+\sigma}-1\right)}\right)\right]}{\chi v_{n} \mu_{n}}\right) ; \\
& j_{25}=-v_{n}(1-\chi)\left(\frac{j_{21} V}{\left(1-\beta_{2}\right) \mu_{n}}+\frac{\xi\left(\beta_{2}-\alpha_{2}\right)}{D}\right) ; \\
& j_{35}=\mu_{X}\left(\xi-\frac{j_{21} V(1-\chi)(\theta-1)}{\theta(1-\sigma)\left(1-\beta_{2}\right) \mu_{n}}\right) ; \\
& j_{45}=\mu_{Q}\left(\xi-\frac{j_{21} V(1-\chi) \alpha_{1}}{\chi\left(1-\beta_{2}\right) \mu_{n}}\right) ; \\
& j_{55}=\frac{\mu_{n}^{-\chi}\left[\mu_{n}^{1+\chi} V \xi(\sigma-1) \chi+A V^{\boldsymbol{\beta}}\left(\chi\left((1-\sigma) \mu_{Q}+\mu_{X}\right)+\mu_{n}(\sigma-1)(1-\chi) \beta_{1}\right)\right]}{V(\sigma-1) \chi} . \\
& \text { where } V^{\boldsymbol{\beta}}=v_{n}^{\beta_{1}} v_{Q}^{\beta_{2}} ; V^{\boldsymbol{\alpha}}=v_{n}^{\alpha_{1}} v_{Q}^{\alpha_{2}} ; V=v_{n} v_{Q} \text {. }
\end{aligned}
$$

